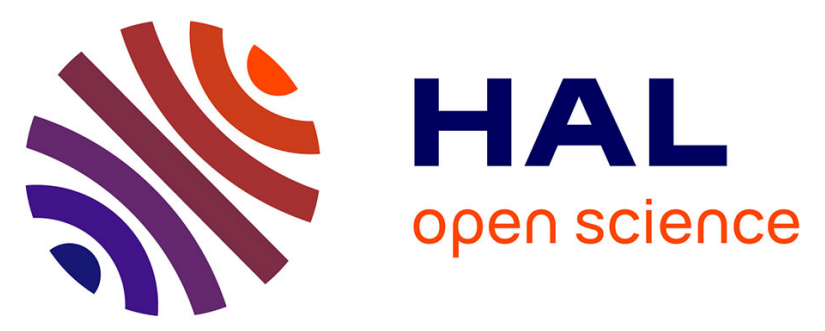

\title{
Modeling for Sustainability
}

Benoit Combemale, Betty H.C. Cheng, Ana Moreira, Jean-Michel Bruel, Jeff Gray

\section{To cite this version:}

Benoit Combemale, Betty H.C. Cheng, Ana Moreira, Jean-Michel Bruel, Jeff Gray. Modeling for Sustainability. Modeling in Software Engineering 2016 (MiSE'16), May 2016, Austin, United States. pp.62-66. hal-01517387v3

\section{HAL Id: hal-01517387 \\ https://inria.hal.science/hal-01517387v3}

Submitted on 3 Mar 2016

HAL is a multi-disciplinary open access archive for the deposit and dissemination of scientific research documents, whether they are published or not. The documents may come from teaching and research institutions in France or abroad, or from public or private research centers.
L'archive ouverte pluridisciplinaire HAL, est destinée au dépôt et à la diffusion de documents scientifiques de niveau recherche, publiés ou non, émanant des établissements d'enseignement et de recherche français ou étrangers, des laboratoires publics ou privés.

\section{(ㄷ)(1) $\Theta$}

Distributed under a Creative Commons Attribution - NoDerivatives| 4.0 International 


\section{Modeling for Sustainability}

\section{Benoit Combemale INRIA and Univ. Rennes 1 \\ INRIA and Univ. Rer
France}

\author{
Betty H.C. Cheng \\ Michigan State University \\ USA
}

\author{
Ana Moreira \\ University Nova de Lisboa \\ Portugal
}

\author{
Jean-Michel Bruel \\ IRIT and Univ. Toulouse \\ France
}

\author{
Jeff Gray \\ University of Alabama \\ USA
}

\begin{abstract}
Various disciplines use models for different purposes. While engineering models, including software engineering models, are often developed to guide the construction of a nonexistent system, scientific models, in contrast, are created to better understand a natural phenomenon (i.e., an already existing system). An engineering model may incorporate scientific models to build a system. Both engineering and scientific models have been used to support sustainability, but largely in a loosely-coupled fashion, independently developed and maintained from each other. Due to the inherent complex nature of sustainability that must balance trade-offs between social, environmental, and economic concerns, modeling challenges abound for both the scientific and engineering disciplines. This paper offers a vision that synergistically combines engineering and scientific models to enable broader engagement of society for addressing sustainability concerns, informed decision-making based on moreaccessible scientific models and data, and automated feedback to the engineering models to support dynamic adaptation of sustainability systems. To support this vision, we identify a number of research challenges to be addressed with particular emphasis on the socio-technical benefits of modeling.
\end{abstract}

\section{INTRODUCTION}

Computer-based systems (CBSs) have contributed significant capabilities and tools needed to address sustainability challenges [6]. Examples include computational modeling, large-scale data analysis, and sensor technology. We use the term sustainability systems to refer to those CBSs that support sustainable development [10], that is, development without a negative global impact. Example sustainability systems include smart grids, smart cities and homes, (climate-)smart agriculture systems, and other CBSs used for resource production, coordination, and management. In contrast, GreenIT refers to those CBSs that have been developed with an explicit intent to minimize resources used

Permission to make digital or hard copies of all or part of this work for personal or classroom use is granted without fee provided that copies are not made or distributed for profit or commercial advantage and that copies bear this notice and the full citation on the first page. Copyrights for components of this work owned by others than the author(s) must be honored. Abstracting with credit is permitted. To copy otherwise, or republish, to post on servers or to redistribute to lists, requires prior specific permission and/or a fee. Request permissions from permissions@acm.org.

MiSE'16, May 16-17 2016, Austin, TX, USA

(C) 2016 Copyright held by the owner/author(s). Publication rights licensed to ACM. ISBN 978-1-4503-4164-6/16/05 . . \$15.00

DOI: http://dx.doi.org/10.1145/2896982.2896992 for development and/or operation (e.g., applications that minimize computational resources). Sustainability systems differ from other types of systems in that their functionality must explicitly balance the trade-offs between the social, economic, and environmental pillars of sustainability [10], thus involving complex decision-making and large volumes of disparate data varying in temporal scale and modality. Sustainability issues have been described primarily by $s c i$ entific models (e.g., mathematical models) that enable scientists to understand the impact of changes in one or more of these three pillars. Engineering models have been used by (software) developers to construct CBSs that support various aspects of sustainability systems, such as ecosystem monitoring, power grid management, and climate-control in smart buildings. This paper proposes a vision that posits that in order to make sustainability systems more accessible and relevant, the scientific models used to understand sustainability concerns must be integrated in a synergistic fashion with the engineering models that are used to support the sustainability systems.

As sustainability concerns gain increasing attention, there is a growing demand for access to sustainability systems by a broader range of stakeholders that have varying technical backgrounds (e.g., community leaders, policy makers, industrial organizations) to make well-informed decisions based on common scientific models. Many of these decisions need to dynamically manage the respective sustainability systems. While progress has been made by leveraging modeling technology to manage the complexity of sustainability systems $[6,3,14]$, numerous software engineering and modeling challenges remain. As the problem complexity, size, and scope increase, stakeholders and their needs change, and technological advances offer new options to exploit. Indeed, the next generation of development approaches should support multiple dimensions of sustainability, ranging from long-lasting dependable and dynamically adaptive software, to green software that requires less computing and fewer energy resources, to software that promotes sustainable, well-informed, human behavior (e.g., smart plugs and appliances, Eco-Batteries to support the home [7]), and market design and regulations that transition consumers towards more energy-saving practices.

Towards this end, several key areas need to be addressed. First, we need to address the challenges of modeling one or more dimensions of sustainability, where we explicitly focus on facilitating the creation and adaptation of models and the respective stakeholders (e.g., scientific, economic, and social) gain increasing understanding of the needs and 
their interaction with other areas. Second, we need to develop technologies to support innovative decision support techniques, integration of disparate data and models coming from different stakeholders, and visualization of model and data integration driven by user inquiries and respective interests [12], all of which must be guided by usability and information access, rather than be limited by traditional computing interfaces for technical wizards. Finally, new development approaches and techniques are needed to tradeoff and cope with the increasing demand of precision, trust, reliability, scalability, adaptation and context-awareness, and timely acquisition and/or computation of information for decision-making.

Guided by these challenges, this vision paper describes the key insights into how modeling can be used to support the development of sustainability systems. Our overall objective is to enable broader engagement of the community, facilitate more informed decision-making, and directly use those decisions to drive the automatic and dynamic adaptation of the sustainability systems.

We observe that many of the foundational concepts used for Model-Driven Engineering (MDE) need to be revisited when considering sustainability systems. Instead of viewing sustainability as yet another application domain, we need to analyze the global nature of such systems to infer the dual and complementary needs of engineering and scientific models. Then, we propose to use a model-driven feedback loop that synergistically uses both the engineering models and the scientific models to support the development and the run-time management of sustainability systems. The engineering models are used to develop and manage the software infrastructure for a CBS for sustainability (e.g., smart grid management). The scientific models are supported by a multi-view scientific modeling infrastructure for capturing the three pillars of sustainability using an Aggregator to integrate multiple scientific models (that is, social and economic models, in addition to the enviornmental models), incorporate monitored data from both the environment and the sustainable system, all of which are used to update the engineering models that (dynamically) manage the sustainable system. The aim is to enable a stakeholder to select specific sustainability "views" to explore (e.g., pose "what if" scenarios across multiple dimensions with "zoom-in" and "zoom-out" capabilities for fine-grained or global-level views, respectively). The results of the science-based inquiries can either be used to predict the impact (e.g., social, economic, or environmental) of a behavior change in resource usage, or used as input to the engineering models to dynamically manage the sustainability system.

The remainder of this paper is organized as follows. Section 2 characterizes the dual and complementary roles of engineering and scientific models. Section 3 introduces our vision of how the two kinds of models are intertwined for the development and run-time management of sustainability systems, and identifies several research challenges posed by this new vision. Finally, Section 4 concludes the paper.

\section{MODELING IN ENGINEERING AND SCI- ENCES}

Engineering models are mainly constructive. Engineers use models to create abstractions of the complex systems under development that do not usually exist at the time the model is built. Several foundational concepts are used by engineering modeling approaches to handle size and tame complexity: decomposition and separation of concerns. MDE can reduce the accidental complexity associated with developing complex software-intensive systems. A primary source of such accidental complexity is the gap between the high-level concepts used by domain experts to express their specific needs and the low-level abstractions provided by general-purpose programming languages [8, 9]. Manually bridging this gap is costly, both in time and effort. MDE approaches address this problem by automatically generating the major system artifacts from models. MDE models focus on how system functionality and domain-specific concepts are modeled relative to how their behavior may be specialized for the respective domain. Domain-specific modeling languages (DSMLs) provide a vocabulary and modeling primitives to facilitate domain experts in developing models describing the intended system behavior [9].

Current MDE approaches used for sustainability systems (such as autonomic and self-adaptive systems) focus on the mechanisms or computing infrastructure (e.g., agent-based systems, self-* systems, architecture models, goal-based models, model-based testing, model-based code generation, modelbased reasoning). The designer of such an adaptive system is faced with a challenging set of modeling and development tasks. One approach to manage the complexity is to use MDE to model the functional system, adaptation logic mechanisms [16], and non-functional properties such as performance and resource use. Model composition and transformation techniques can be leveraged to support automated generation of the target sustainability system [14, 11]. Depending on the specific MDE approach used, models can be created manually, automatically created based on requirements specifications, or a combination thereof.

Given the inherent uncertainty with these systems, both from the environment and from the modeled systems, it is difficult, if not impossible, to anticipate all possible conditions that will occur at run-time [4]. One approach is to use models at run-time to manage the system [2], including self-healing and reconfiguration, and maintaining consistency between the changing system and the corresponding models [17] relative to specific environmental contexts [1]. In this case, the data provided by the sensors are automatically fed back to the engineering models in order to adapt the system at run-time.

Either used at design-time or at run-time, engineering models focus on the system itself and its interactions with the environment (e.g., communication with sensors and actuators). However, the interplay with broader information coming from scientific models (e.g., analysis and predictive models), regulations (e.g., economic, social and environmental laws) and user models (e.g., preferences, strategic choices, etc.) still pose many modeling challenges.

Scientific models are largely descriptive. Scientists also use modeling to handle the complexity of the phenomena under study. Of course, to be useful for communication, models have to delicately balance abstraction with concrete information, where assumptions on the world must be made explicit and communicated in a language that can be understood by stakeholders [13]. A causal connection must be made between the models and the real-world to ensure the fidelity of the result when attempting to understand the phenomena. The principle of substitution, often associated with 
simulation, is important in this context so that scientists have confidence in the model query results. Scientific models are used to explain and predict the behavior of real-world phenomenon, which led the philosopher K. Popper ${ }^{1}$ to the characterization of scientific theories as falsifiable models. Scientists abstract away complex details, and typically the models they construct only apply when considered within certain boundaries that are of interest. Abstraction always means that certain properties are omitted while (hopefully) the relevant ones, with respect to the purpose of the model, are captured in sufficient detail to fulfill the model's purpose. Whether a model is useful can therefore only be answered with the knowledge about its purpose.

Recently, we conducted an empirical study to evaluate the effectiveness of using DSMLs to support the simulation of a water management system for use in a farming context [3]. DSMLs were effective in engaging the domain experts in modeling both topological and behavioral concerns. Our investigation showed that by using DSMLs to raise the level of abstraction of modeling to domain-specific concepts (rather than programming elements), we were able to broaden the use of MDE to a range of domain experts working with the water management system. From the positive feedback generated by these investigations, we identified additional points that warrant further study in order to make the experience of adding abstraction through the use of modeling techniques even more beneficial to the modeling process and the resulting analysis capabilities. Areas for further work include improving tool usability, with customized support for modeling and data analysis strategies that are more in line with the domain user's scientific inquiry process and perception of the system model; providence of the data and model. The main conclusion of the work was that the current trends in MDE are progressing in the right direction with increasing support for modeling and managing complex systems.

\section{SOCIO-TECHNICAL MODELING SHIFT}

Computing and technology continue to play an increasing role to support sustainability research. More sophisticated ecosystem monitoring techniques have yielded petabytes of data for scientists to analyze. As scientists build increasingly more complex scientific models, additional challenges are posed to the computing disciplines to make the data and model-based analysis results more accessible and understandable to the scientists and other stakeholders, as well as its integration into the engineering models for use in the corresponding sustainability systems.

Figure 1 depicts an integrated approach where each model provides feedback to the other. In this approach, engineering models are dynamically adapted by interpreting the resulting impact on the sustainability system, while scientific models are impacted by the use of the engineering models in a feedback loop that continuously adapts the system to reflect the tradeoffs and changes in priorities among the three pillars. Other stakeholders (e.g., individuals, community leaders, policy makers, industrial organizations) can select specific (personalized) views of sustainability to explore the impact of changes in social behavior, policies, and resource consumption (cf. Model Experiencing Environments - MEEs - to support "what-if" scenarios [12]).

\footnotetext{
${ }^{1}$ See http://plato.stanford.edu/entries/popper/
}

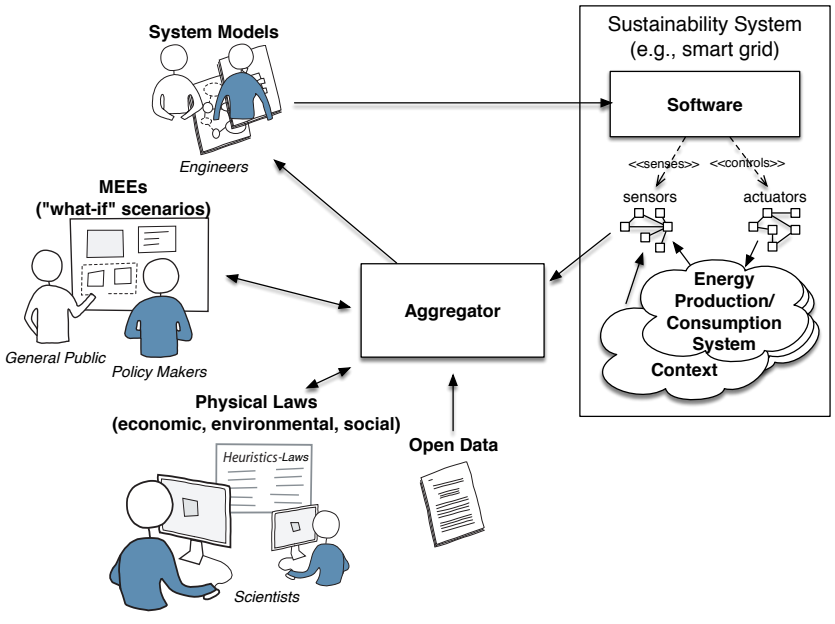

Figure 1: Intertwined use of engineering and scientific models

At the core of the approach is the Aggregator concept. Its objective is threefold: i) to ensure the fusion of the various heterogeneous data sources (scientific models, open data, personal expectations, and information collected from the sensors); ii) to adapt the scientific models accordingly and to provide specific views accessible to a broader public; and iii) to (automatically) update the engineering models according to science-informed decisions taken from the scientific models, open data and (personal or community) preferences. Next, we elaborate each of the three objectives and finish by raising additional orthogonal challenges.

\subsection{Model and data fusion to support informed decision}

A sustainability system requires consideration of the many trade-offs between the numerous scientific models when looking for potential solutions, where solutions may involve changes to one or more of the three pillars of sustainability, as well as the sustainability system itself. For example, when considering power consumption and power production from multiple sources (e.g., wind, solar, nuclear, water), policy analysts may use the Aggregator to explore several scenarios involving the respective scientific models and the sensors from the sustainability systems to determine what to do during a drought season with record high temperatures. The Aggregator analysis may suggest how to adjust cost models and/or legislate temporary laws during the drought season (e.g., impose fines for washing cars or watering lawns). In addition, the feedback from the Aggregator to the smart grid system may be to harness more power from solar sources, decrease demand from water-based sources, and reroute power to high-demand areas to ensure that hospitals and other care facilities have sufficient resources to run air-conditioning, among other needs.

\subsection{Personalizing sustainability for broader en- gagement}

A key insight to our vision is that sustainability approaches must be user-centric, focusing on individuals' interests, back- 
ground, and objectives. According to OPOWER ${ }^{2}$, "What's at stake are tomorrow's energy consumers. To thrive for another century, utilities must capture their attention and exceed their expectations. It starts with the customer experience." To effectively address sustainability, the customer has to understand and share the vision of the providers, and vice versa. Previously, we proposed the concept of Model Experiencing Environments (MEEs) [12] as an approach to support complex model and data integration, while offering customizable interfaces for accessing model analysis results and their visualizations. In this paper, the Aggregator is intended to support the different types of model and data integration needed by a MEE, where a MEE interface and visualization support is customizable according to a given user and his respective interests. The objective of MEEs is to enable different types of stakeholders to "experience" models according to their perspectives and level of interests and needs. A MEE can be used as an educational medium for enabling children and others to learn about the impact of their individual actions and decisions (e.g., what is the impact of taking 4-minute showers instead of 15-minute showers over a year long period on water consumption for the household? for the town?). MEEs can also be used to study the effects of collective behavior by a community (e.g., what is the impact on environmental resources by decreasing red meat consumption down to once per week?). For land use or environmental policy analysts, MEEs can be used to pose more sophisticated "what if?" scenarios to understand the impact of specific policies or legislation. MEEs empower users to better understand the cause and effects of individual and global actions and decisions. MEEs aim at making the complexity of the scientific models, the data and model fusion process, the distributed nature of the data, and the resolution of temporal and spatial differences between the models transparent to the MEE user, thus enabling them to focus on the science questions.

\subsection{Feedback to engineering models for dy- namic adaptation}

Each of the social, economic and environmental pillars must have their respective information captured in a (domainspecific) analysis and predictive models. New integration techniques are needed to support a systematic, well-defined approach to integrate these disparate models to enable wellinformed decisions and functionality to be provided by sustainability systems. This push furthers the challenge of the globalization of modeling languages [5], to support a technical coordination between the scientific and engineering models into the control loop of adaptive systems. When the Aggregator is integrating the scientific models, it must consider the temporal and spatial dependencies, as well as the granularity of the model and sensor data from the sustainability system. Updates to the engineering models for the sustainability system must consider the type of user engaged with the system. That is, an individual power consumer will not be allowed to effect change to the power distribution system. But the management of a power generation company may use the MEE interface to explore different "what if?" scenarios to determine the most reliable and cost-effective strategy for delivering power to their customers. The results of the analysis may propose dynamic updates to the sustainability system via the feedback link from the Aggregator.

\subsection{Additional Socio-Technical Concerns}

Several additional areas need to be considered to facilitate the socio-technical modeling shift. Approaches should include resource usage analytics techniques to enable researchers to examine complex relationships between models and variables, using the power of predictive analytics to understand behavior patterns and the impact of one pillar with respect to the other pillars. New approaches should integrate ideas from behavioral science to produce persuasive solutions to engage individuals; therefore, going beyond the traditional one-size-fits-all solutions. Such a scenario may involve thousands or millions of people, data security, and anonymity to preserve customer privacy, all of which need to be planned from the very early stages of development. Finally, the proposed models, languages and techniques need to be able to handle the voluminous amount of disparate data coming from a wide variety of sources. In the era of Big Data, reducing large-scale problems to a scale (along multiple dimensions, such as information volume, visualization, and software complexity) that humans can comprehend and act upon is fundamental [15].

\section{CONCLUSIONS}

This paper addresses the role of modeling for sustainability systems. We are convinced that models will play an essential role in integrating complex information and promoting a broader engagement of various stakeholders into the control loop of adaptive systems responsible for specific sustainability concerns (e.g., resource management systems).

We examine how models are used by engineers and scientists. While both communities have made great advances in modeling ever more complex problems, and also increased the sophistication of how models can be used, analyzed, and executed, we postulate that effectively using the modeling efforts from both communities in a more coordinated and collaborative fashion offers the potential to address important challenges in the development of sustainability systems.

In this paper we envision an approach where MDE is used to support both the integration of the various heterogeneous models through a unified modeling framework, and the socio-technical coordination through the use of different, specifically tailored, DSMLs. The social coordination is intended to support the involvement and collaboration of various stakeholders (e.g., engineers, scientists, decision makers, general public), and the technical coordination is intended to ensure the consistency and impact analysis between the various models as well as the automatic consideration into the control loop of adaptive systems.

To achieve this vision, we identify several key challenges for the MDE community, including the integration of heterogeneous models, the need for personalizing specific views and domain-specific concepts for different stakeholders, and the automatic adaptation of engineering models from scientific models. Finally, we discuss the necessity to integrate both large scale simulation of analysis models and probabilistic analysis of predictive models.

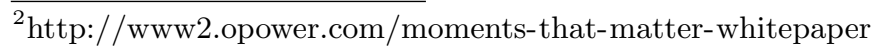




\section{REFERENCES}

[1] M. Acher, P. Collet, F. Fleurey, P. Lahire, S. Moisan, and J.-P. Rigault. Modeling Context and Dynamic Adaptations with Feature Models. In MRT'09, 2009.

[2] N. Bencomo. On the use of software models during software execution. In MISE'09. IEEE, 2009.

[3] J.-M. Bruel, B. Combemale, I. Ober, and H. Raynal. $\mathrm{MDE}$ in Practice for Computational Science. In ICCS, 2015.

[4] B. Cheng, A. Ramirez, and P. McKinley. Harnessing evolutionary computation to enable dynamically adaptive systems to manage uncertainty. In $C M S B S E$, pages 1-6. IEEE, 2013.

[5] B. Combemale, J. DeAntoni, B. Baudry, R. B. France, J. Jézéquel, and J. Gray. Globalizing modeling languages. IEEE Computer, 47(6):68-71, 2014.

[6] Committee on Computing Research for Environmental and Societal Sustainability. Computing Research for Sustainability. National Academies Press, 2012.

[7] J. Dean. Power to the people in energy revolution: Eco-batteries will slash household bills. The Times, May 2015.

[8] R. France and B. Rumpe. Model-driven Development of Complex Software: A Research Roadmap. In FOSE'0\%. IEEE, 2007.

[9] J. Gray, J.-P. Tolvanen, S. Kelly, A. Gokhale, S. Neema, and J. Sprinkle. Domain-specific modeling. In Handbook of Dynamic System Modeling, pages 1-20. CRC Press, 2007.

[10] S. Imperatives. Report of the World Commission on Environment and Development: Our Common Future.

[11] B. Morin, F. Fleurey, N. Bencomo, J.-M. Jézéquel, A. Solberg, V. Dehlen, and G. Blair. An aspect-oriented and model-driven approach for managing dynamic variability. In MODELS'O8.

[12] G. Mussbacher, D. Amyot, R. Breu, J.-M. Bruel, B. Cheng, P. Collet, B. Combemale, R. France, R. Heldal, J. Hill, J. Kienzle, M. Sch"ottle, F. Steimann, D. Stikkolorum, and J. Whittle. The Relevance of Model-Driven Engineering Thirty Years from Now. In MODELS'14. Springer.

[13] A. Quarteroni. Mathematical models in science and engineering. Notices of the AMS, 56(1):10-19, 2009.

[14] B. Solomon, D. Ionescu, M. Litoiu, and M. Mihaescu. Model-driven engineering for autonomic provisioned systems. In COMPSAC'08.

[15] J. Thomas and K. Cook, editors. Illuminating the Path: Research and Development Agenda for Visual Analytics. IEEE Press, 2005.

[16] T. Vogel, S. Neumann, S. Hildebrandt, H. Giese, and B. Becker. Model-driven architectural monitoring and adaptation for autonomic systems. In ICAC'09. ACM.

[17] T. Vogel, S. Neumann, S. Hildebrandt, H. Giese, and B. Becker. Incremental model synchronization for efficient run-time monitoring. In Models in Software Engineering, volume 6002 of $L N C S$, pages 124-139. Springer Berlin Heidelberg, 2010. 